\title{
A dimensão da amígdala cerebral e a agressividade no touro de lide
}

\author{
Gouveia, A.J., ${ }^{1,2,} ;$ Orge, L. ${ }^{3,4}$ e Carvalho, P. ${ }^{3}$
}

'Instituto Politécnico de Portalegre. C3i. Portalegre. Portugal.

${ }^{2}$ Instituto Nacional de Investigação Agrária e Veterinária. Oeiras. Portugal.

${ }_{3}^{3}$ Instituto Nacional de Investigação Agrária e Veterinária. Lisboa. Portugal.

${ }^{4}$ Universidade de Trás-os-Montes e Alto Douro. CECAV. Vila Real. Portugal.

\section{PALAVRAS CHAVE ADICIONAIS}

Bovino.

Raça Brava de Lide.

Encéfalo.

Anatomia cerebral.

Sistema límbico.

\section{ADDITIONAL KEYWORDS}

\section{Bovine.}

Bullfighting breed.

Encephalon.

Brain anatomy.

Limbic system.

\section{RESUMO}

Estudos anátomo-fisiológicos implicaram zonas cerebrais como o córtex orbitofrontal e pré-frontal, áreas do hipocampo e a amígdala, em particular, no controlo do comportamento agressivo em animais e em humanos. Este estudo de parametrização do encéfalo e da amígdala cerebral em bovinos de raça Brava de Lide, em comparação com bovinos cruzados de aptidão de carne, teve por objetivo verificar a existência de uma possível associação entre aquela área cerebral e o comportamento agressivo manifestado pelos touros de lide. Os autores avaliaram a morfologia e as dimensões do encéfalo e da amígdala cerebral em 120 bovinos, 60 da raça Brava de Lide e 60 cruzados produtores de carne, e correlacionaram a dimensão da amígdala cerebral com o comportamento dos touros de lide nas corridas à Portuguesa. Constatou-se que os 2 grupos de bovinos analisados apresentaram diferenças na conformação dos hemisférios cerebrais, na relação peso do encéfalo/peso da carcaça em quente e na dimensão da amígdala cerebral. Nos touros de lide, as amígdalas cerebrais apresentaram menores dimensões, de uma forma estatisticamente muito significativa, evidenciando-se também uma separação nítida entre touros com adequada aptidão para lide, que apresentavam amígdalas menores, e aqueles cujo comportamento foi impróprio para a lide. Esta diferença anatómica poderá contribuir para a diferente modulação do comportamento apresentado.

\section{The size of amygdala and aggressiveness in fighting bulls}

\section{SUMMARY}

Aggressive behaviour control of both animals and humans seems to be dependent on several brain areas, such as the orbitofrontal and prefrontal cortex, part of the hippocampus and the amygdala. This study evaluated the morphology and size of the brain, as well as fighting bulls' amygdala in comparison to beef cattle, in order to verify a putative association of this brain structure with aggressive behaviour modulation in fighting bulls. The authors assessed the morphology and dimensions of the brain and amygdala in 120 bovines, 60 fighting bulls and 60 crossbred beef cattle, and correlated the size of the amygdala with the behaviour presented by fighting bulls during Portuguese bullfighting. The two groups showed differences concerning cerebral hemispheres conformation, brain weight/carcass weight ratio and amygdala size. The latter was remarkably smaller in fighting bulls than in beef cattle. Moreover, it was demonstrated that fighting bulls with a smaller amygdala size presented a higher bullfighting ability than those showing an inappropriate behaviour for bullfighting. Thus, this anatomical feature may contribute to the different modulation of behavioural patterns displayed.

\section{INFORMACIÓN}

\section{Cronología del artículo.}

Recibido/Received: 17.08.2015

Aceptado/Accepted: 09.11.2015

On-line: 16.03 .2016

Correspondencia a los autores/Contact e-mail:

prof.augustogouveia@gmail.com

\section{INTRODUÇÃO}

O bovino de Raça Brava de Lide é um expoente de agressividade condicionada, a qual apresenta especial particularidade de ser repetitiva até à exaustão. Existem vários fatores, alguns bem documentados, que contribuem parcialmente para a explicação da agressividade nesta raça, nomeadamente os genéticos (Cossio,
2007; Illera et al., 2007; Mateus e Russo-Almeida, 2014) e os baixos níveis de serotonina (Cabrera, 2013; Cabrera et al., 2013). No entanto, na avaliação de qualquer comportamento também devem ser considerados outros aspetos como a neuroanatomia e a neurofisiologia. Quanto ao efeito de determinadas estruturas anatómicas no comportamento exibido pelos touros de lide, 
os estudos são muito limitados. Na década de 70, José Delgado, Professor de Fisiologia em Yale, testou um touro de lide, verificando um possível envolvimento da amígdala cerebral (Rev. por Horgan, 2005).

Noutras espécies, como o gato e o macaco, a indução e a perceção de estímulos elétricos no hipotálamo e amígdala foram claramente correlacionados com alterações de comportamento (Rhawn, 2000). Nestas espécies, quando a amígdala é ativada eletricamente, o hipotálamo é também ativado e as reações agressivas são despoletadas. Algumas investigações em primatas não-humanos têm demonstrado que danos na amígdala neonatal induzem uma reatividade emocional anormal perante objetos e parceiros sociais; quando confrontados com uma ameaça potencial, macacos adultos com lesões na amígdala expressaram menos medo e maior agressividade perante a possível ameaça (Rev. por Raper et al., 2013).

De igual modo no homem, amígdalas menores têm sido consideradas o fator mais comum nos indivíduos que praticaram crimes violentos (Siegel e Victoroff, 2009), embora haja também envolvimento do córtex orbitofrontal que regula a atividade da amígdala, influenciada ainda pelos esteroides sexuais (Almeida et al., 2015). Estes aspetos anatómicos bem como disfunções nestas regiões neuroanatómicas têm sido observados na conduta violenta humana, no transtorno de personalidade antissocial (Serrano et al., 2014), nos serial killers (Moon, 2013) e nos psicopatas (Moskowitz, 2011), bem como em indíviduos com histórias de violência sofridas na infância (Varella, 2011). Numa outra linha de investigação, foram medidas as amígdalas cerebrais a indivíduos que se voluntariaram, de acordo com um protocolo previamente estabelecido: os voluntários com níveis mais altos de agressão exibiram uma redução de 8 -16 \% no volume da amígdala, verificando-se assim uma correlação negativa altamente significativa entre os volumes da amígdala versus agressão (Matthies et al., 2012).

Embora não seja crível que o tamanho do encéfalo inteiro, per se, seja uma variável útil se o quisermos relacionar diretamente com o comportamento, há um considerável mérito na análise da variação e tamanho das partes do encéfalo, uma vez que algumas dessas zonas podem ser relacionadas com funções específicas (Healy e Rowe, 2007). Como estes estudos têm vindo a implicar a amígdala no controlo da agressividade nos animais e no homem, a parametrização do encéfalo e da amígdala constituiu o principal objetivo deste trabalho preliminar para uma eventual correlação entre a dimensão da amígdala e o comportamento observado nos bovinos de raça Brava de Lide, os quais exibem uma agressividade repetida e intensa sem treino prévio. Deste modo, procedeu-se à morfometria do encéfalo e da amígdala cerebral em bovinos desta raça e à sua comparação com bovinos produtores de carne, bem como à verificação de uma possível relação das dimensões encontradas, com o comportamento apresentado por aqueles animais durante a lide em praça.

\section{MATERIAL E MÉTODOS}

Entre 2013 e 2014, os encéfalos analisados foram colhidos, no Matadouro Regional de Mafra, de forma aleatória, de acordo com a cadeia de processamento e desman-cha, sem interferir com a produtividade dos trabalhos inerentes. Um total de 120 encéfalos foi colhido - 60 de bovinos machos da raça Brava de Lide (RBL), com mé-dia de idade de 55 meses e de 60 bovinos produtores de carne (BPC), maioritariamente cruzados de Limousine ou Charolês com a raça Alentejana. As colheitas nestes bovinos, todos do sexo masculino, foram efetuadas em animais com idade igual ou superior a 18 meses (média de 23 meses), considerando para estes híbridos precoces o estado fisiológico de adulto. Devidamente identificados e acondicionados foram transportados para o Instituto Nacional de Investigação Veterinária (INIAV) onde se procedeu ao registo do peso e à mensuração do encéfalo nos sentidos crânio-caudal e latero-lateral (ao nível dos lobos temporais). Após um corte transversal ao nível do tálamo, efetuado sistematicamente a $1 \mathrm{~cm}$ caudalmente ao quiasma ótico (Kommadath et al., 2011), as amígdalas foram observadas macroscopicamente e medidos os seus 2 eixos: altura e largura, dada a sua forma ovóide. As pesagens e as medições, efetuadas com uma régua paquímetro, foram realizadas com os encéfalos a fresco. Adicionalmente, numa secção transversa ao nível dos núcleos da base, a dimensão do córtex cerebral foi medida desde a superfície dorsal até ao limite dorsal do corpo caloso nos dois grupos de bovinos.

Apresentando a amígdala cerebral uma forma elíptica, foi possível determinar a sua área a partir das medidas dos eixos $2 a$ (maior) e $2 b$ (menor). Numa elipse -o conjunto de pontos de um plano cujas somas das distâncias a dois pontos fixos desse plano é uma constante- a área é determinada pela fórmula: $\pi \cdot a / 2 . b / 2$ (Da Silva, 2014).

Todos os dados registados foram analisados através do software SPSS Versão 12 complementado com a folha de cálculo Microsoft Office Excel 2013 com Statistical Analysis Plug-In to Microsoft Excel (Harnett e Horrel, 1998), onde se estabeleceram tanto para a RBL como para os BPC, as médias, máximos, mínimos e o desvio padrão, bem como a elaboração de diagramas de extremos e quartis (Boxplot) comparativos, concluindo para um intervalo de confiança (IC) de $95 \%$ os respetivos intervalos e aferindo assim, os graus de significância, com apresentação das respetivas transposições gráficas. O tratamento estatístico foi realizado através do teste $t$-Student, o qual é utilizado para verificar a plausibilidade da hipótese nula ser verdadeira (diferença igual a zero) ou não e assim aferir-se sobre a dissemelhança de médias entre grupos. Esta premissa é normalmente usada quando a estatística do teste, na verdade, segue uma distribuição normal, mas a variância da população $\sigma^{2}$ é desconhecida, então, é usada a variância amostral $S^{2}$ e, com esse ajuste, a estatística do teste passa a seguir a distribuição t-Student (Raju, 2005). A quantificação da amostra para a análise estatística foi determinada a partir de um cálculo simples, que pode ser usado quando a dimensão da população é conhecida, a qual refere que a dimensão de uma amostra proveniente de uma população finita, de tamanho $\mathrm{N}$ é dado por: $\mathrm{N}^{(1 / 2)}+1$ arredondado para o número inteiro mais próximo (Arsham, 2015). Deste modo, a população - conhecida e finita de touros de lide 
corridos em praças portuguesas em 2013-foi de 1538 (Lucas, 2014), de que resultou numa amostragem de 40 animais aceites para o estudo contudo, e ainda assim, foram estudados mais 20, oriundos de um total de 24 ganadarias. Usando o mesmo método de amostragem, foi solicitado posteriormente à Associação Portuguesa de Criadores de Touros de Lide (APCTL), através do Secretário-Técnico do Livro genealógico da raça Brava de Lide, a informação valorativa do comportamento em lide, de 9 touros dos 60 analisados. No entanto, foram ainda escolhidos aleatoriamente mais 6 touros, perfazendo um total de 15 (correspondendo a $25 \%$ da amostra inicial), dos quais 5 apresentaram amíg-dalas menores $(<0,5 \mathrm{~cm} 2), 5$ com dimensões médias $(0,6$ a 0,7 $\mathrm{cm} 2)$ e os restantes com dimensões elevadas $(>0,8$ $\mathrm{cm} 2$ ). A lista enviada apenas continha os touros com o número do sistema de identificação animal à frente da qual se classificaram os touros com boas condições de lide usando: $\mathrm{R}$ - Regular (atitudes típicas de bravo, cumprindo em todas as sortes - 4 pontos); B

- Bom (bravos com investidas permanentes -6 pontos) e MB - Muito bom (bravos com ferocidade e investidas de longe - 8 pontos). Aos touros sem condições de lide foi-lhes atribuída a classificação de M - Mau (atitudes de defesa, refúgio, investidas curtas - 2 pontos).

$\mathrm{Na}$ análise valorativa do comportamento em lide elaborou-se um gráfico composto, permitindo assim relacioná-lo com as áreas das amígdalas.

\section{RESULTADOS}

Na conformação externa dos hemisférios cerebrais, nos bovinos da raça Brava de Lide (RBL) verificou-se que os polos rostrais ou frontais eram mais estreitos até aproximadamente ao nível da fossa lateral, contrastando com os polos caudais ou occipitais que são mais volumosos e arredondados [figura 1(a)], lembrando um cone de extremo romboide. Nos bovinos produtores de carne (BPC), os hemisférios cerebrais apresentam ambos os polos arredondados [figura 1 (b)]. Na avaliação do córtex cerebral ao nível dos núcleos da base, constatou-se que a superfície era menos convexa nos bovinos RBL [figura 1 (c)]. Na figura 1 (d), está indicada a conformação ovóide das amígdalas cerebrais num bovino desta raça.

No que concerne aos pesos encontrados nos encéfalos, tanto na RBL como nos BPC, encontrámos variações assinaláveis que se traduziram numa diferença de $75 \mathrm{~g}$ nas médias simples entre ambos.

Não sendo possível determinar a área do encéfalo, as medidas expressas na tabela I, que equivalem respetivamente aos eixos crânio-caudal (comprimento) e latero-lateral (largura) obtidos em cada grupo de bovinos foram relacionados entre si para identificar o grau da maior ou menor relação estabelecida, tendo-se verificado, através do teste $t$-Student, que em ambos os casos e grupos as médias obtidas não são significativamente diferentes. Foi ainda determinada uma relação comprimento/largura $(\mathrm{C} / \mathrm{L})$ para a RBL de 1,00 e de 1,02 para os BPC, mas estes valores não são significativamente diferentes em termos estatísticos, confirmando as medidas simples que lhe deram origem. A avaliação do peso do encéfalo com o peso corporal só
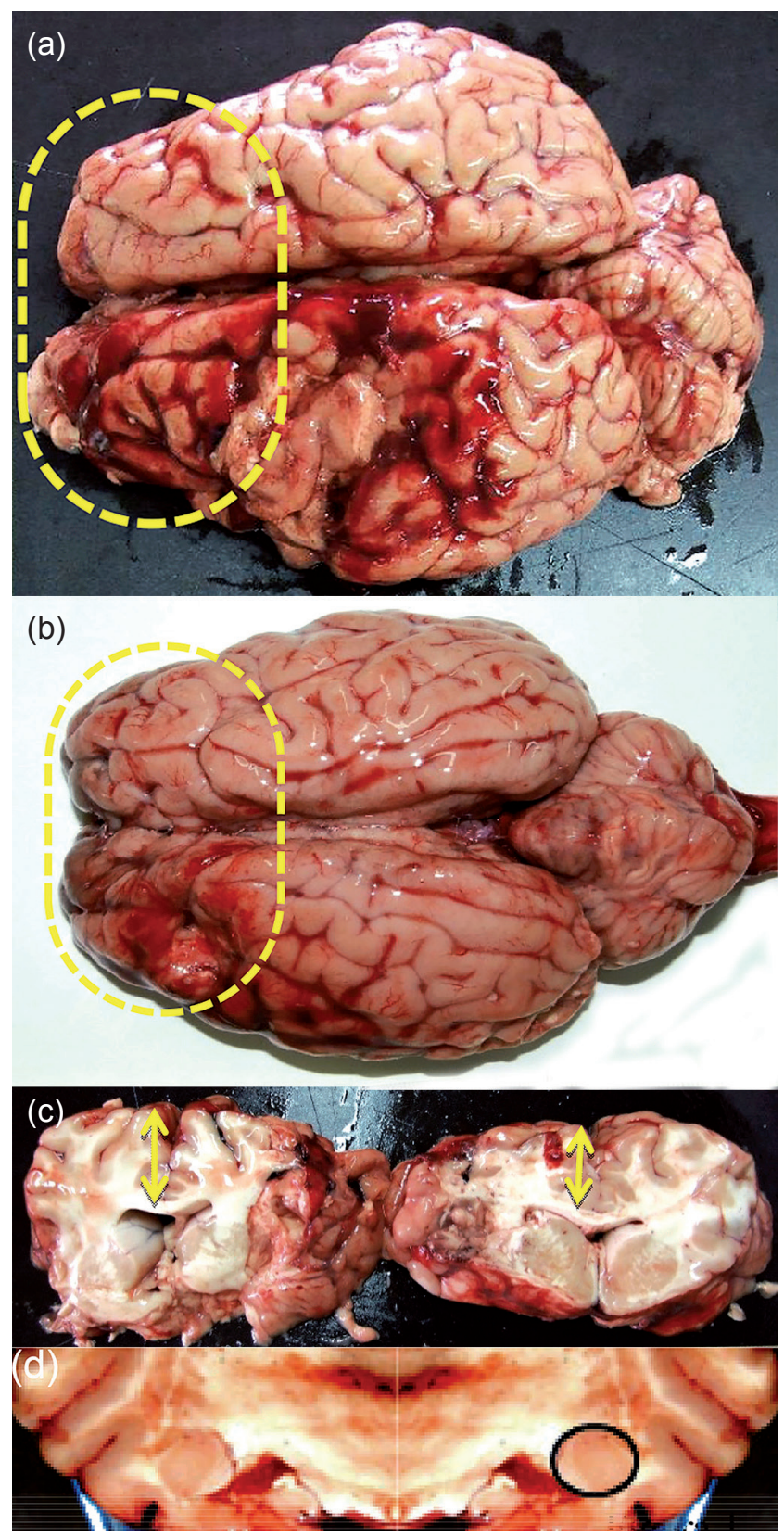

Figura 1. Aspetos morfológicos do encéfalo de bovinos analisados (Morphological aspect of analyzed bovine's encephalon). (a) Bovino de raça Brava de Lide (RBL) apresentando os polos frontais estreitos (área assinalada a amarelo) e os polos occipitais mais largos (encéfalo com peso $408 \mathrm{~g}$, eixo crânio-caudal $11 \mathrm{~cm}$ e eixo latero-lateral 11,3 cm); (b) Bovino produtor de carne (BPC) com os polos frontais arredondados (área assinalada a amarelo) e com uma largura mais próxima dos polos occipitais (encéfalo com peso 438 g, eixo crânio-caudal $11 \mathrm{~cm}$ e latero-lateral $11 \mathrm{~cm}$ ); (c) Altura do córtex cerebral ao nível dos núcleos da base (setas) maior no cérebro do BPC (cérebro da esquerda) $(2,7 \mathrm{~cm})$ quando comparado com o da RBL $(2,4 \mathrm{~cm})$ (BPC- encéfalo 366 g; eixo crânio-caudal 11,4 $\mathrm{cm}$; eixo latero-lateral 10,5 cm; RBL-350 g; 9,8 cm e $10,2 \mathrm{~cm}$ para os mesmos eixos); (d) Visualização das amígdalas cerebrais nos dois hemisférios cerebrais (amígdala direita delimitada a preto) (dimensões 0,9 $\mathrm{cm}$ de largura e $0,7 \mathrm{~cm}$ de altura) de um bovino de raça Brava de Lide (encéfalo com $447 \mathrm{~g} ; 10,7 \mathrm{~cm}$ e 11,4 cm para os eixos crânio-caudal e latero-lateral, respetivamente). 
Tabela I. Parametrização dos encéfalos - RBL e BPC (Encephalon parameterization - RBL and BPC).

\begin{tabular}{lcccc}
\hline & \multicolumn{2}{c}{ RBL } & \multicolumn{2}{c}{ BPC } \\
\hline$(\mathrm{cm})$ & $\mathrm{A}$ & $\mathrm{L}$ & $\mathrm{A}$ & $\mathrm{L}$ \\
\hline Média & 10,967 & 10,968 & 10,877 & 10,738 \\
Máximo & 15,000 & 12,100 & 12,300 & 12,300 \\
Mínimo & 8,1000 & 0,900 & 9,400 & 8,7000 \\
Desvio padrão & 0,9632 & 0,6041 & 0,7043 & 0,7152 \\
IC (95\%) & 11,$21 ; 10,71$ & 11,$12 ; 10,81$ & 11,$05 ; 10,69$ & 10,$92 ; 10,53$
\end{tabular}

$\mathrm{RBL}=$ Raça Brava de Lide; $\mathrm{BPC}=$ Bovino produtor de carne; $\mathrm{A}=\mathrm{An}-$ chura $\mathrm{L}=\mathrm{Largura} ; \mathrm{IC}=$ intervalo de con iança.

Tabela II. Relação: Peso do encéfalo/peso carcaça $(Q)$ - RBL e BPC (Relation: Encephalon weight/carcass weight (Q) $\mathrm{RBL}$ and $\mathrm{BPC}$ ).

\begin{tabular}{lcc}
\hline & Raça Brava de Lide & Bovino produtor de carne \\
\hline Média & 1,434 & 1,236 \\
Máximo & 2,878 & 2,397 \\
Mínimo & 0,724 & 0,588 \\
Desvio Padrão & 0,401 & 0,372 \\
IC (95\%) & 1,$53 ; 1,33$ & 1,$33 ; 1,13$
\end{tabular}

$\mathrm{RBL}=$ Raça Brava de Lide; $\mathrm{BPC}=$ Bovino produtor de carne; $\mathrm{IC}=$ intervalo de confiança.

Tabela III. Parametrização das amígdalas cerebrais RBL e BPC (The parameterization of cerebral amygdala - RBL and $B P C$ ).

\begin{tabular}{lcccc}
\hline & \multicolumn{2}{c}{ Raça Brava de Lide } & \multicolumn{2}{c}{ Bovino produtor de carne } \\
\hline$(\mathrm{cm})$ & $\mathrm{L}$ & $\mathrm{A}$ & $\mathrm{L}$ & $\mathrm{A}$ \\
\hline Média & 0,8367 & 1,1433 & 0,8417 & 1,8633 \\
Máximo & 1,2000 & 2,4000 & 1,0000 & 2,6000 \\
Mínimo & 0,5000 & 0,5000 & 0,6000 & 1,2000 \\
Desvio padrão & 0,1288 & 0,4260 & 0,1119 & 0,2314 \\
IC (95\%) & 0,$87 ; 0,80$ & 1,$25 ; 1,03$ & 0,$87 ; 0,81$ & 1,$92 ; 1,80$ \\
\hline
\end{tabular}

$\mathrm{RBL}=$ Raça Brava de Lide; $\mathrm{BPC}=$ Bovino produtor de carne; $\mathrm{A}=\mathrm{An}$ chura; L= Largura; IC= intervalo de con iança.

é rigorosa se partirmos do peso individual vivo. No entanto, apenas tivemos acesso aos pesos das carcaças em quente $(\mathrm{Q})$, cuja correspondência ao peso vivo só pode ser feito mediante estimativa inversa, sendo este rendimento em carcaça muito variável entre bovinos da mesma raça e idade. $\mathrm{O}$ rendimento da carcaça em quente, atribuído ao touro de lide, tem por base um estudo efetuado durante a Feira de San Isidro (Madrid) em 2002, onde foram lidados a cavalo 146 touros, 19 novilhos e 18 reses, obtendo-se um rendimento de $55,6 \%$ (Fuente et al., 2003). Assim, efetuaram-se as inter-relações entre os pesos dos encéfalos e das carcaças em quente $(Q)$ de que resultaram os dados obtidos na tabela II e representados em gráfico sob a forma de diagrama de extremos e quartis (figura 2). Estes resultados expressam que as médias dos valores encontrados são diferentes, apresentando um nível estatisticamente muito significativo de $1 \%(* *)$. A mediana na RBL é de 1,37 e nos BPC é de 1,16. Na percentagem

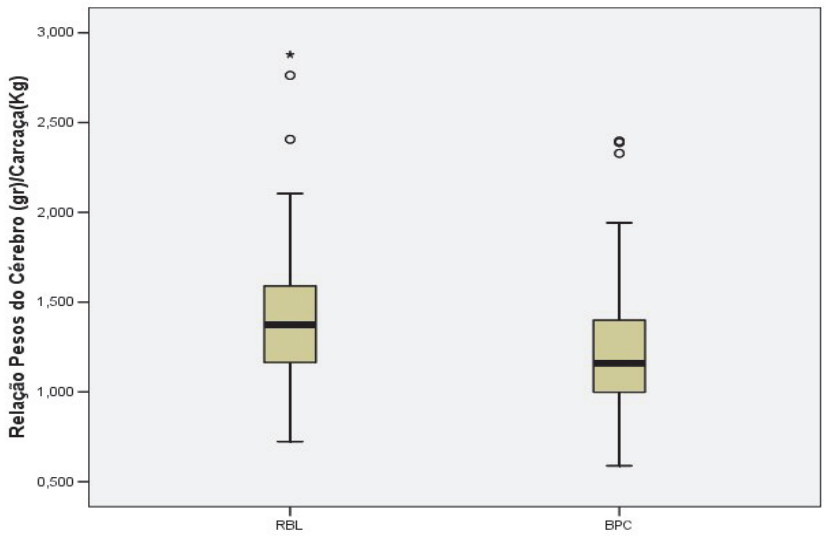

Figura 2. Diagrama comparativo da relação entre os pesos do encéfalo $(\mathrm{g})$ e da carcaça em quente $(\mathrm{Kg})$ na RBL e nos BPC (Comparison of the relationship between the weight of encephalon ( $\mathrm{g}$ ) and hot carcass ( $\mathrm{kg}$ ) in RBL and BPC). (RBL= Raça Brava de Lide; $B P C=$ Bovino produtor de carne).

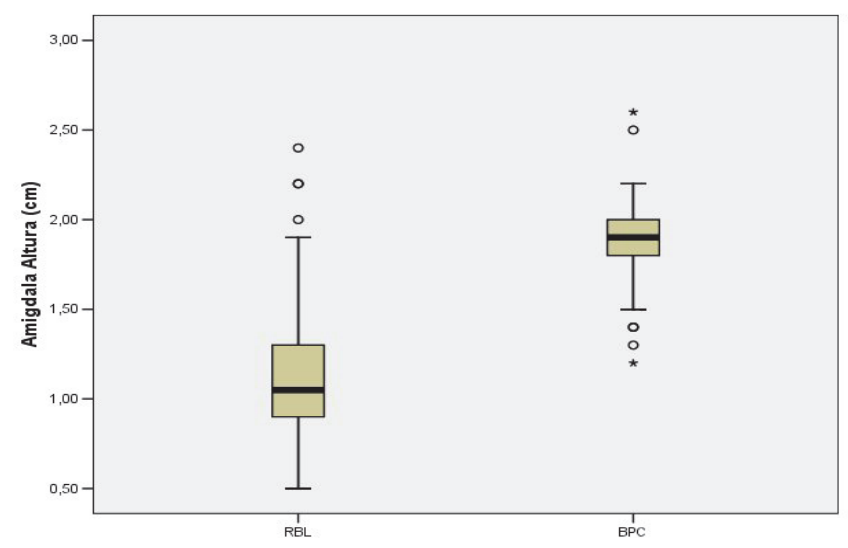

Figura 3. Diagrama comparativo do parâmetro da altura das amígdalas - RBL e BPC (Comparative parameter of amygda-lae's height - RBL and BPC). $(\mathrm{RBL}=$ Raça Brava de Lide; $\mathrm{BPC}=$ Bovino produtor de carne).

do peso do encéfalo em relação ao peso da carcaça (Q), os resultados expressam uma diferença nas médias dos valores encontrados a um nível estatisticamente muito significativo de $1 \%\left(^{* *}\right)$. A mediana na RBL é de $13,7 \%$ e os BPC obtêm 11,6\%. De facto, os dados reais obtidos corroboram o modelo, dado que a RBL e os BPC têm em média uma relação peso de encéfalo/peso de carcaça $(\mathrm{Q})$ respetivamente de $1 / 700(0,14 \%)$ e de $1 / 850$ $(0,12 \%)$, isto é, ainda que o encéfalo da RBL tenha em média 353 g e os BPC tenham $428 \mathrm{~g}$, o peso da carcaça em quente de ambos, em média, é de $258 \mathrm{~kg}$ (RBL) e $370 \mathrm{~kg}$ (BPC).

Os resultados das medidas colhidas na medição direta das amígdalas (largura e altura) em $\mathrm{cm}$ estão indicados na tabela III. As médias encontradas para a largura na RBL e nos BPC não são significativamente diferentes. A mediana da largura na RBL é de 0,9 e nos BPC 0,8 . No entanto, para o parâmetro da altura, dada a inusitada relevância que assumiu, foram estes dados convertidos em diagrama de extremos e quartis (figura 3), cujos resultados, expressam médias diferentes para um nível estatístico altamente significativo $\left.0,1 \%{ }^{* * *}\right)$. As medianas da altura têm na RBL e nos $\mathrm{BPC}$, respetivamente os valores de 1,05 e de 1,9. 
Tabela IV. Amplitudes de dados nos parâmetros analisados - RBL e BPC (Data amplitude of the analyzed parameters- $\mathrm{RBL}$ and $\mathrm{BPC}$ ).

\begin{tabular}{lcccc}
\hline & \multicolumn{2}{c}{$\mathrm{RBL}$} & \multicolumn{2}{c}{$\mathrm{BPC}$} \\
\cline { 2 - 5 } & $\mathrm{M}$ & $\mathrm{m}$ & $\mathrm{M}$ & $\mathrm{m}$ \\
\hline Área da amígdala $\left(\mathrm{cm}^{2}\right)$ & 1,3 & 0,4 & 1,7 & 0,7 \\
Encéfalo relação $\mathrm{C} / \mathrm{L}$ & 1,5 & 0,79 & 1,38 & 0,84 \\
Peso encéfalo $(\mathrm{g})$ & 463 & 223 & 538 & 331 \\
Peso carcaça $\mathrm{Q}(\mathrm{kg})$ & 361 & 131 & 677 & 179 \\
Relação peso-encéfalo/carcaça-Q & 0,29 & 0,07 & 0,24 & 0,06 \\
Idade (meses) & 203 & 32 & 89 & 18 \\
\hline
\end{tabular}

$\mathrm{RBL}=$ Raça Brava de Lide; $\mathrm{BPC}=$ Bovino produtor de carne; $\mathrm{M}=$ Máximo; m= Mínimo.

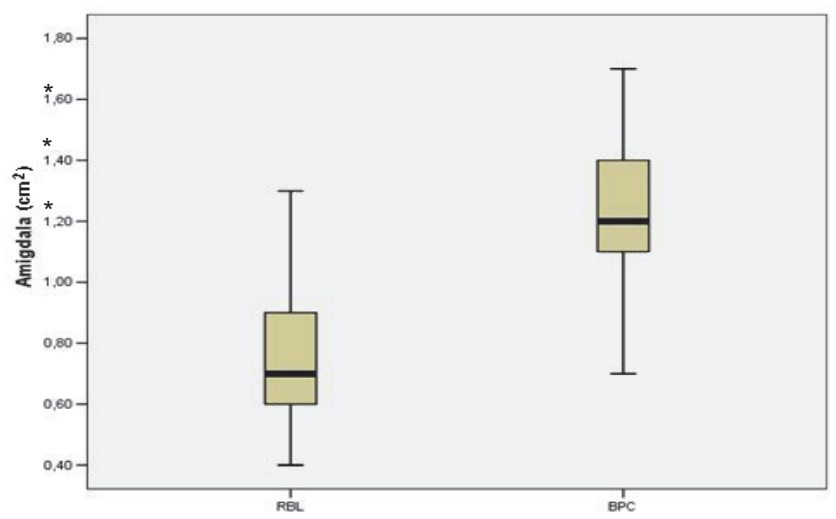

Figura 4. Diagrama comparativo das áreas das amígdalas na RBL e nos BPC (Comparison of the amygdalae areas in RBL and $\mathrm{BPC})$. (BL= Raça Brava de Lide; $\mathrm{BPC}=$ Bovino produtor de carne).

Determinou-se a área (oval) da amígdala cerebral tanto na RBL como nos BPC, obtendo-se assim as dimensões médias para ambos os grupos. Contudo, para além dos valores absolutos inferiores encontrados na RBL, foi fundamental saber se esta menor dimensão seria ou não, estatisticamente significativa. Os resultados obtidos nas áreas das amígdalas de ambos os grupos expressam que as médias dos valores encontradas são diferentes, para um nível estatístico altamente significativo de $0,1 \%{ }^{* * *}$ ) (figura 4 ). A mediana na RBL tem $0,7 \mathrm{~cm}^{2}$ e nos BPC tem $1,2 \mathrm{~cm}^{2}$. Da relação de valores encontrados nos parâmetros determinados, de acordo com a abordagem do nosso estudo, resultaram os dados apresentados na tabela IV (RBL e BPC). A partir dos dados obtidos não foram encontradas correlações lineares significativas entre os vários parâmetros, não se conseguindo explicar no modelo os valores observados, tanto na RBL como nos BPC, sejam os da amígdala, da relação comprimento/largura do encéfalo, do peso do encéfalo, do peso da carcaça em quente (Q), do \% peso do encéfalo vs peso da carcaça, ou a idade. Assim, foi efetuada a estatística tendo por base o teste t-Student para médias de amostras independentes e o uso do diagrama de extremos e quartis (Boxplot). Tendo por base os dados recolhidos de forma sistemática e padronizada, os resultados comparativos dos tratamentos estatísticos obtidos, entre a RBL e os BPC, são os constantes na tabela V.

Relativamente à classificação do comportamento dos 15 bovinos de RBL em lide nas Praças com Corri- das à Portuguesa, verificou-se que 10 animais (67\%) apresentavam condições de lide, dos quais um com comportamento de muito bom, dois com bom e os restantes sete que mereceram a classificação de regular. Os restantes cinco (33\%) não apresentaram condições

Tabela V. Estatística de resultados dos parâmetros analisados-RBL e BPC (Statistical results of the analyzed parameters $-\mathrm{RBL}$ and $\mathrm{BPC}$ ).

\begin{tabular}{lcccc}
\hline & \multicolumn{2}{c}{$\mathrm{RBL}$} & \multicolumn{2}{c}{$\mathrm{BPC}$} \\
\cline { 2 - 5 } & $\mathrm{M}$ & $\mathrm{IS}$ & $\mathrm{M}$ & IS \\
\hline Área da amígdala $\left(\mathrm{cm}^{2}\right)$ & 0,7 & 0,$78 ; 0,68$ & 1,2 & 1,$29 ; 1,17$ \\
Encéfalo relação $\mathrm{C} / \mathrm{L}$ & 352,9 & 363,$5 ; 342$ & 428 & 438,$5 ; 417,5$ \\
Peso encéfalo $(\mathrm{g})$ & 1,00 & 1,$03 ; 0,97$ & 1,02 & 1,$04 ; 1,00$ \\
Peso carcaça Q $(\mathrm{kg})$ & 258 & 271,$4 ; 244,8$ & 370 & 394,$8 ; 346,2$ \\
Relação peso-encéfalo/ & 0,14 & 0,$154 ; 1,133$ & 0,12 & 0,$133 ; 0,114$ \\
carcaça-Q & 54,5 & 60,$4 ; 48,7$ & 22,7 & 25,$7 ; 17,3$ \\
ldade (meses) & & & &
\end{tabular}

$(\mathrm{RBL}=$ Raça Brava de Lide; $\mathrm{BPC}=$ Bovino produtor de carne $)$. $M=$ Média; IS=Intervalo de segurança.

Tabela VI. Classificação do comportamento de touros de lide (nas corriḑas à Portuguesa) (Fighting bulls behaviour classification (in Portuguese bullfighting)).

\begin{tabular}{|c|c|c|}
\hline Identificação & Classificação & Amígdala cerebral Área $\left(\mathrm{cm}^{2}\right)$ \\
\hline PT- - - - - - 5 & Muito Bom & 0,4 \\
\hline PT- - - - - 0 & Bom & 0,7 \\
\hline PT- - - - - 0 & Bom & 0,7 \\
\hline PT- - - - - 5 & Regular & 0,4 \\
\hline PT- - - - - 6 & Regular & 0,4 \\
\hline PT- - - - - 8 & Regular & 0,4 \\
\hline PT- - - - - 2 & Regular & 0,5 \\
\hline PT- - - - - 4 & Regular & 0,6 \\
\hline PT- - - - - 6 & Regular & 0,7 \\
\hline PT- - - - - 4 & Regular & 0,7 \\
\hline PT- - - - - - 7 & Mau & 0,8 \\
\hline PT- - - - - 7 & Mau & 0,8 \\
\hline PT- - - - - 0 & Mau & 0,9 \\
\hline PT- - - - - 7 & Mau & 0,9 \\
\hline PT- - - - - 2 & Mau & 1,0 \\
\hline
\end{tabular}

$\mathrm{PT}=$ Portugal

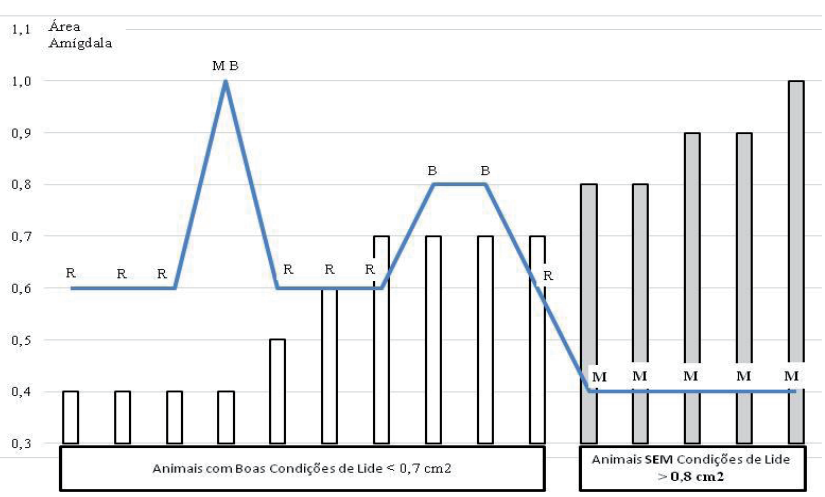

Figura 5. Relação das dimensões das amígdalas com a classificação do comportamento da RBL nas corridas de touros à portuguesa (Relationship of the dimensions of amygdalae with the RBL behavior classification in the Portuguese bullfighting). (RBL= Raça Brava de Lide). 
aceitáveis de lide devido a características de mansidão (tabela VI). As dimensões das amígdalas que determinámos, e que se encontram entre 0,4 e $0,7 \mathrm{~cm}^{2}$, correspondem a touros adequados às lides e, portanto, com um desempenho de bravo, expectável na Raça Brava de Lide. A maior dimensão da amígdala $\left(>0,8 \mathrm{~cm}^{2}\right)$ determinou, na análise ao comportamento em lide, os touros com alterações de comportamento atípicas, mais relacionadas com os bovinos mansos (figura 5).

\section{DISCUSSÃO}

As dimensões médias do encéfalo encontradas nos 2 grupos de bovinos (RBL e BPC) são maiores do que as descritas para a espécie bovina por Barone e Bartolami (2004) (comprimento 10,5 cm; largura 10,4 cm), podendo este fato estar relacionado com o método de medição. Relativamente ao peso médio do encéfalo nos dois grupos de bovinos, este foi menor ( $R B L=353 \mathrm{~g}$; $\mathrm{BPC}=428 \mathrm{~g}$ ) do que o indi-cado pelos mesmos autores (510 g, com mínimo - $400 \mathrm{~g}$ e máximo - $700 \mathrm{~g}$ ). No entanto, a proporção do peso do encéfalo com o peso corporal de $1 / 700$ e de $1 / 850$ na RBL e BPC, respetivamente, está de acordo com o descrito pelos mesmos autores.

Embora a domesticação esteja relacionada com uma redução de 25 - $34 \%$ do tamanho do encéfalo em re-lação às formas silvestres, conforme foi constatado em algumas espécies, particularmente das ordens Carnivo-ra e Artiodactyla (Rev. por Kruska, 1996), no presente estudo as medidas e a relação comprimento/largura do encéfalo não foram significativamente diferentes nos dois grupos.

Na comparação dos 2 grupos, encontraram-se diferenças significativas nos parâmetros analisados:- a conformação do cérebro, a dimensão da amígdala cerebral e o peso do encéfalo/peso da carcaça em quente (Q).

Observaram-se diferenças significativas nos hemisférios cerebrais, quer ao nível dos polos frontais quer na convexidade da superfície dorsal, evidenciando os bovinos RBL uma estrutura mais estreita daqueles polos e um adelgaçamento do córtex cerebral que pode justificar essa superfície menos convexa. De acordo com Pérez-Barbería e Gordon (2005), ungulados que vivem em grupo e que estão constantemente sujeitos a interações sociais apresentaram encéfalos maiores; no entanto, Shultz e Dunbar (2006) concluíram que as exigências cognitivas de sociabilidade estão mais relacionadas com o tamanho do cortex cerebral. Este facto pode explicar a diferença encontrada aquando da mensuração do cortex cerebral na RBL e nos BPC.

No que tange às áreas das amígdalas cerebrais, estas evidenciaram menores dimensões na RBL, de uma forma estatisticamente muito significativa, quando comparada com as dos BPC. Se considerarmos para os BPC a média de uma amígdala de base 1, então teríamos para a RBL uma amígdala $42 \%$ menor. $\mathrm{Na}$ RBL, também se verificou uma relação negativa muito proeminente e significativa entre a dimensão da amígdala e a agressividade. Esta afirmação tem por base o comportamento observado durante a lide dos touros identificados na amostra, dos quais se deduziu uma clara separação entre touros com adequada ap- tidão para a lide (amígdalas $<0,5 \mathrm{~cm}^{2}$ ) e aqueles cujo comportamento foi impróprio para a lide (amígdalas $>0,8 \mathrm{~cm}^{2}$ ) devido a atitudes de manso. Embora não seja possível estabelecer um paralelismo com os vários estudos desenvolvidos em humanos, que aliam uma menor dimensão da amígdala cerebral ao incremento da violência (Siegel e Victoroff, 2009; Moskowitz, 2011; Varella, 2011; Matthies et al., 2012; Moon, 2013), não deixa de ser significativa a similaridade observada na Raça Brava de Lide. Todavia, não se poderá inferir que um touro de lide com uma amígdala com uma área superior a $1,3 \mathrm{~cm}^{2}$ (valor máximo encontrado), tenha um comportamento desviante. Nos touros de lide, a agressividade assenta em múltiplas variáveis contributivas e atribuíveis ao meio ambiente, à fisiologia e à genética maioritariamente (Illera et al., 2007).

Estudos dos núcleos que compõem a amígdala cerebral, o tipo de neurotransmissores e a expressão de recetores serão necessários para aprofundar a avaliação do papel desta estrutura neuroanatómica.

\section{CONCLUSÃO}

No cumprimento do objetivo a que nos propusemos, consideramos que a recoleção de dados obtidos e aferida técnica e cientificamente foi demonstrativa, apresentando um nível estatístico altamente significativo nas mensurações comparativas. Este trabalho de morfo-parametrização permitiu concluir que a amígdala cerebral nos bovinos da Raça Brava de Lide é menor que a dos bovinos produtores de carne. Também se constatou uma relação entre a dimensão da amígdala e agressividade observada em lide, deduzindo-se uma clara separação entre touros com adequada aptidão para a lide (amígdalas $<0,7 \mathrm{~cm}^{2}$ ) e aqueles cujo comportamento foi impróprio (amígdalas $>0,8 \mathrm{~cm}^{2}$ ).

\section{AGRADECIMENTOS}

Os autores agradecem aos que contribuíram e permitiram a execução deste estudo.

Doutor Miguel Fevereiro, Coordenador da Unidade Estratégica de Investigação e Serviços de Produção e Saúde Animal (I.S.P.S.A.)- INIAV.

Dr. Eurico Esteves, Médico Veterinário e Administrador do Matadouro Regional de Mafra.

Eng. ${ }^{\circ}$ Agrónomo, MSc. Vitor Conceição Martins INIAV (Instituto Nacional de Investigação Agrária e Veterinária).

Dr. Vasco Lucas, Médico Veterinário e Secretário Técnico do Livro Genealógico da Raça Brava de Lide - Associação Portuguesa de Criadores de Touros de Lide (APCTL).

\section{BIBLIOGRAFIA}

Almeida, R.M.M.; Cabral, J.C.C. and Narvaes, R. 2015. Behavioral, hormonal and neurobiological mechanisms of aggressive behavior in human and nonhuman primates. Physio/ Behav, 143: 121-135. http:// www.sciencedirect.com/science/article/pii/S0031938415001316 (24/05/2015). 
Arsham, H. 2015. Statistical thinking for managerial decisions. In: Dr. Arsham's statistics site. Edition online by Dr. Hossein Arsham. http:// home.ubalt.edu/ntsbarsh/business-stat/opre504.htm (28/04/2015).

Barone, R. et Bartolami, R. 2004. Anatomie comparé des mammifères domestiques, 6. Neurologie I. Système Nerveux Central. Editions Vigot Frères. Paris. França.

Cabrera, F.G. 2013. Variables neuroendocrinas y su relación con el comportamiento durante la lidia del toro bravo, (Bos taurus, L.). Tesis Doctoral. Universidad Complutense de Madrid. Facultad de Veterinaria. España. http://eprints.ucm.es/17868/ (25/04/2015).

Cabrera, F.G.; Gema, S.; Gonzalez, A.G. e Illera, A.G. 2013. Neuroendocrinología del comportamiento agresivo en el Toro de Lidia. Comunicación en: VII Symposium del Toro de Lidia. Zafra.

Cossio, J.M. 2007. Los toros. Tratado técnico y histórico. Tomo I. Espasa Calpe. Madrid. España.

Da Silva, J.P. 2014. Como calcular a área e o perímetro de uma elipse? http://www2.fc.unesp.br/revistacqd/v3n1//v3n1_art1.pdf (19/06/2015).

Fuente, D.; Guerra, J.; Ortuño, S.; Fernández, C.; Durán, J.M.; Ballesteros, C. y Vigo, M. 2003. Estudio del peso a la canal de los animales lidiados en la Plaza de Toros de Las Ventas durante la temporada de 2002. Comunicación en el VI Symposium del Toro de Lidia. Zafra. España. http://www.centrotorolidia.es/opencms_wf/opencms/investigacion/3_ponencias/todos/ponencia_0088.html (28/04/2015).

Harnett, D.L. and Horrel, J.F. 1998. Statistical analysis plug-in to Microsoft Excel Version 5.0. Kaddstat Multimedia CD. Wiley. New York. USA.

Healy, S.D. and Rowe, C. 2007. A critique of comparative studies of brain size. P Roy Soc B, 274: 453-464. http://rspb.royalsocietypublishing. org/ (28/04/2015).

Horgan, J. 2005. The work of José Delgado, a pioneering star. The forgotten era of brain. Sci Am. hHtp://www.scientificamerican.com/ article/the-forgotten-era-of-brai/?print=true (28/04/2015).

Illera, J.C.; Gil, F. y Silván, G. 2007. Regulación neuroendocrina del estrés y dolor en el Toro de Lidia (Bos taurus L.): Estudio preliminar. RCCV, 2: 1-6.

Kommadath, A.; Woelders, H.; Beerda, B.;Mulder, H.; Wit, A.; Veerkamp, A.C.; Pas, F.P. and Smiths, M.A. 2011. Gene expression patterns in four brain areas associate with quantitative measure of estrous behavior in dairy cows. Bio Med Cent Genomics, 12: 200. http://www. biomedcentral.com/1471-2164/12/200 (20/06/2015).

Kruska, D. 1996. The effect of domestication on brain size and composition in the mink (Mustela vison). J Zool, 239: 645-661.

Lucas, V. 2014. Informações anotadas. Secretário Técnico do Livro Genealógico dos Bovinos de Raça Brava de Lide. Associação Portuguesa de Criadores de touros de lide (APCTL). Porto Alto. Samora Correia. Matthies, S.R.; Weber, N.; Lieb, M.; Philipsen, K.; Tuescher, A.; Ebert, O.; Hennig, D.J. and Van Elst, J.L.T. 2012. Small amygdala - High aggression? The role of the amygdala. Modulating aggression in healthy subjects. World JBiol Psychiatry, 13:75-81. http://informahealthcare. com/doi/abs/10.3109/15622975.2010.541282 (28/04/2015).
Mateus, J.C. and Russo-Almeida, P.A. 2014. Exploring the genetic diversity and substructure of the Portuguese cattle breed Brava de Lide using microsatellites. FAO. Anim Genet Resour, 55: 9-17. http://journals. cambridge. org/action/displayAbstract? fromPage=online\&aid $=9450$ 582\&fileld=S2078633614000149 (28/04/2015).

Moon, T. 2013. Serial killers have under-developed brains. http://www.ibtimes.co.uk/criminal-psychology-neoroscience-adrian-raine- 459614 (20/04/2015).

Moskowitz, C. 2011. Criminal minds are different from yours, brain scans reveal. Neuroscience and psychology of criminal minds and psychopaths. http://www.livescience.com/13083-criminals-brainneuroscience-ethics.html (01/05/2015).

Pérez-Barbería, F.J. and Gordon, I.J. 2005. Gregariousness increases brain size in ungulates. National Institute of Health. Oecologia Australis (AO), 145: 41-52. http://www.ncbi.nlm.nih.gov/pubmed/16032436 (01/05/2015).

Raju, T.N. 2005. William Sealy Gosset and William A. Silverman: two students of science. Pediatrics, 116: 732-735.

Raper, J.; Wilson, M.; Sánchez, M.; Machado, C.J. and Bachevalier, J. 2013. Pervasive alterations of emotional and neuroendocrine responses to an acute stressor after neonatal amygdala lesions in rhesus monkeys. Psychoneuroendocrinology, 38: 1021-1035. www.elsevier. com/locate/psyneuen (01/05/2015).

Rhawn, J. 2000. Critical Issues in neuropsychology, neuropsychiatry, and behavioral neurology. Fear, rage and aggression, improving memory, amygdala. Ed. Academic Press. New York. J Neuropsychiatry Clin Neurosci. https://books.google.pt/books?hl=pt-PT\&lr=\&id=VBpwlY gHgkkC\&oi=fnd\&pg=PA1 \&dq=Rhawn, + J.+(2000) + +Fear, + Rage + an $d$ +aggression,+Improving+Memory,+Amygdala.+Neuropsychiatry, +Neuropsychology,+Clinical+Neuroscience.+(Academic+Press,+New +York).\&ots=ocOpPitjJ\&\&sig=kl qFpJpofbk5AO_hirzl-ROu_vQ\&redir_ esc $=y \# v=$ onepage\&q\&f=false $(01 / 05 / 2015)$.

Serrano, M.A.S.; Villamil, E.; Salas, H.J.E.M.; Castro, S.G. y Sapudio, LL. 2014. Revisión conceptual de los sustratos neuropsicológicos y psicológicos asociados á la conducta violenta humana. Rev Neuropsicol Latinoam SLAN, 6: 31-40. http://www.neuropsicolatina.org/index. php/Neuropsicologia_Latinoamericana/article/viewFile/142/148 (03/05/2015).

Siegel, A. and Victoroff, J. 2009. Understanding human aggression: New insights from neuroscience. Int J Law Psychiat, 32: 209-215. http:// www.sciencedirect.com/science/article/pii/S0160252709000661 (03/05/2015).

Shultz, S. and Dunbar, R.I.M. 2006. Both social and ecological factors predict ungulate brain size. P Roy Soc B, 273: 207-215.

Varella, D. 2011. Raízes biológicas da violência. Neurociência. Faculdade de Medicina da Universidade de S. Paulo. http://www.scribd.com/ doc/73875865/Textos-Sobre-Violencia-de-Drauzio-Varella\#scribd (24/04/2015). 
